\title{
The GAP Portion of Pseudomonas Aeruginosa Type III Secreted Toxin ExoS Upregulates Total and Surface Levels of Wild Type CFTR
}

\author{
Deepali N. Tukaye Sang-Ho Kwon Wiliam B. Guggino \\ Department of Physiology, Johns Hopkins University School of Medicine, Baltimore, MD
}

\author{
Key Words \\ ExoS-GAP • CFTR • Pseudomonas aueroginosa
}

\begin{abstract}
Background: Pseudomonas aeruginosa (PA) infections account for a large percentage of fatal hospital acquired pneumonias. One of the PA Type III secreted toxin (TTST) ExoS, a bifunctional protein with $\mathrm{N}$-terminal GTPase activating protein (GAP) and C-terminal ADP rybosyl transferase (ADPRT) activities, significantly contributes to PA virulence by targeting small molecular weight G-proteins (SMWGP). In this study, we have looked at one of the mechanisms by which the GAP portion of ExoS (ExoS-GAP) mediates cellular toxicity. Methods: The effects of ExoS-GAP on CFTR trafficking were studied in CFBE410- Kir 2.2 and MDCK cell lines stably expressing CFTR using a transient transfection system. Results: Transient transfection of ExOS-GAP increased the total and surface protein levels of mature wild type CFTR in epithelial cells stably expressing wild type (WT) CFTR. The effect of ExoS-GAP was specific to CFTR in bronchial epithelial cells since it did not affect the total protein levels of $\mathrm{Na}^{+} / \mathrm{K}^{+}$ATPase, another membrane protein. A point mutation in the ExoS GAP domain (R146K), known to disrupt its catalytic GAP activity, abolished the effect of ExoS-GAP on WT CFTR. Lysosomal inhibition studies with Bafilomycin A1 indicate that ExoS-GAP decreased lysosomal degradation of the mature WT CFTR with concomitant increase in the total levels of mature WT CFTR. However, ExoS-GAP did not increase the total protein levels of $\triangle$ F508CFTR. Conclusion: The GAP portion of the PA TTST ExOS increases the total and surface levels of wild type CFTR in vitro mammalian cell system. The effect of ExoS-GAP on WT CFTR total protein levels provides new insight into understanding the virulent pathophysiology of PA infections.
\end{abstract}

Copyright $@ 2013$ S. Karger AG, Basel

\section{Introduction}

Pseudomonas aueroginosa (PA) infections are commonly encountered in hospitalized or immune compromised individuals [1,2]. In its acute form, PA infections present as fulminant pneumonias [1]. PA is a leading cause of nosocomial pneumonia with $>50 \%$ mortality despite 
medical management [1]. The clinical pathology of PA pneumonia includes pulmonary fluid exudates rich in protein with bacteremia [3].

PA pathogenesis is mediated by an arsenal of virulence factors, which include a variety of toxins. Of these, the PA Type III secreted toxins (TTST), ExoU, ExoY, ExoS and ExoT, have been widely studied [4]. These toxins possess motifs with enzymatic activities, which account for their pathogenesis [4]. ExoS is a bifunctional protein with an N-terminal GTPase activating protein (GAP) domain and a C-terminal ADP ribosyl transferase domain (ADPRT) [5]. ExoS modulates the activity of a variety of small molecular weight G proteins (SMWGP) and its effects on Rho, Rac1, and Cdc42 are well known[4, 6-8]. The GAP domain has been shown to disrupt cell-cell adhesion by disrupting the actin cytoskeleton mediated by the inhibition of Rho family SMWGP [7]. A variety of Rabs are involved in the trafficking of protein cargo, including membrane proteins, between various cellular compartments[9].

CFTR, a 12 transmembrane domain ABC transporter [10], is a vital chloride channel in the lungs. It localizes to the apical membrane of airway epithelial cells and is responsible for the generation of airway surface fluid and the maintenance of mucociliary clearance $[10,11]$. Alteration in the expression or function of CFTR dramatically affects these processes and hence the innate airway defense [11-13]. The first extracellular loop of CFTR is believed to be the binding region for the PA surface lipopolysaccharide (LPS) [13]. This CFTR-mediated cellular uptake of PA has been postulated to be an important mechanism for clearing the organism from airway surface epithelium [13]. It has been shown that PA lung infections stimulate an increase in the surface levels of CFTR in mice $[13,14]$. Also, in the mouse model of Pseudomonas pneumonia, the lungs of mice infected with PA ExoS ${ }^{+}$strains are significantly heavier than those of mice infected with PA ExoS- strains resulting from increased fluid content in the lungs [15]. However, the molecular mechanisms underlying these observations are not well understood.

Targeting of CFTR by bacterial toxins is a proposed pathophysiological basis for infection by numerous bacteria including Vibrio cholera [16], Salmonella typhi [17], Pseudomonas aueroginosa $[13,14]$. PA expresses a number of toxins of which the Type III Secreted Toxins (TTST) contribute to significant virulence [18-20]. PA strains expressing the TTST ExoS are found in majority of clinical PA pneumonia isolates; $72 \%$ isolates in one study [21]. ExoS is also shown to be a prominent virulence factor promoting bacterial dissemination of PA infections in the burned mouse model [22-24]. The GAP activity of ExoS is known to inactivate Rab family SMWGP [25]. A number of Rabs are involved in the trafficking of CFTR from the Golgi to the plasma membrane, at the plasma membrane (Rab5, Rab4, and Rab11) and to the lysosome (Rab7, Rab9) $[9,10]$. Based on these observations, it is logical to conceptualize that ExoS-GAP contributes to pathology by altering the cellular trafficking of various proteins. Our experiments have focused on understanding effects of the GAP activity of ExoS (ExoSGAP) on CFTR in epithelial cells.

Identifying molecular mechanisms underlying the pathophysiology of PA pneumonia would provide insight into the fulminant nature of the disease and development of newer treatment strategies. In the present study, we have explored the effect of the ExoS GAP domain on the trafficking of CFTR in epithelial cells. Our data indicates that the GAP activity of ExoS increases the total and surface levels of mature WT CFTR. We also show that the increase in protein levels can be attributed to decreased lysosomal degradation of mature WT CFTR by the GAP activity of ExoS.

\section{Materials and Methods}

\section{Cell culture}

Human cystic fibrosis bronchial epithelial cells, CFBE41o- Kir 2.2 cells, provided by Dr. Dieter Gruenert, express stably transfected WT CFTR. CFBE cells were grown in minimum essential medium (MEM; Invitrogen/Gibco Cat. \# 11095-080) containing 10\% FBS, L-glutamine (2mM final concentration), penicillin \& streptomycin (500 $\mu \mathrm{g} / \mathrm{ml}$ and $50 \mu \mathrm{g} / \mathrm{ml}$ respectively) and hygromycin $(300 \mu \mathrm{g} / \mathrm{ml}$; Invitrogen 
Tukaye/Kwon/Guggino: ExoS-GAP Upregulates WT CFTR

Cat.10687-010). Madin-Darby canine kidney (MDCK) epithelial cells, provided by Dr. Stanton, express stably transfected GFP-tagged WT CFTR. MDCK cells are grown in Dulbecco's modified eagle medium (DMEM; Invitrogen/Gibco Cat. \# 11095-080) medium containing 10\% FBS, L-glutamine (2mM final concentration), penicillin \& streptomycin $(500 \mu \mathrm{g} / \mathrm{ml} \& 50 \mu \mathrm{g} / \mathrm{ml}$ respectively) and hygromycin $(200 \mu \mathrm{g} / \mathrm{ml}$; Invitrogen Cat.10687-010).

\section{Constructs}

The full length ExoS construct with mutated ADPRT activity (ExoS- E381D-HA), provided by Dr. Joseph T. Barberi, is HA-tagged for use in biochemical toxin detection. The construct and resulting protein are referred to as ExoS-GAP. The R146K point mutation was generated by using the Stratagene QuickChange Site-Directed Mutagenesis kit (\#200518 and \#200519). The following primers were generated (by Invitrogen) to introduce a single R to K mutation at position 146 in the ExoS construct- Forward: 5'- cca gcg gag atg ggg cgc tga agt cgc tga gca ccg cct tgg c - 3'; Reverse: 5'- gcc aag gcg gtg ctc agc gac ttc agc gcc cca tct ccg ctg g -3'. PCR reactions were carried out using $5 \mu \mathrm{l}$ of $10 \mathrm{X}$ reaction buffer, $10 \mathrm{ng}$ of ExoS plasmid, 125 ng of each primer, $1.5 \mu \mathrm{l}$ of dNTP mix, $1 \mu \mathrm{l}$ of Pfu Turbo DNA polymerase and double-distilled $\mathrm{H}_{2} \mathrm{O}$ to give a final volume of $50 \mu \mathrm{l}$. Reaction mixtures were cycled as follows: 1 cycle at $95^{\circ} \mathrm{C}$ for $30 \mathrm{sec} ; 18 \mathrm{cycles}$ of $95^{\circ} \mathrm{C}$ for $30 \mathrm{sec}, 55^{\circ} \mathrm{C}$ for $1 \mathrm{~min}, 68^{\circ} \mathrm{C}$ for $1 \mathrm{~min}$ per kb of plasmid length. The PCR product was treated with $1 \mu \mathrm{l}$ of DpnI restriction enzyme, gently mixed, centrifuged for $1 \mathrm{~min}$ and the reaction mix was incubated at $37^{\circ} \mathrm{C}$ for $1 \mathrm{hr}$. A $100 \mu \mathrm{l}$ aliquot of XL1-Blue supercompetent cells was transformed with $1 \mu \mathrm{l}$ of DpnI treated DNA from the reaction mixture. Transformed XL1-Blue supercompetent cells were incubated on LB-Kanamycin agar plates at $37^{\circ} \mathrm{C}$ overnight. Isolated colonies were seeded into $2 \mathrm{ml}$ of $\mathrm{LB}$ broth and cultured at $37^{\circ} \mathrm{C}$ overnight; plasmid extraction was performed using a Quiagen Miniprep Kit and the plasmid was sequenced at the Johns Hopkins DNA core. The plasmids with appropriate sequence were used to transform competent cells (DH5 $\alpha$ ) for plasmid amplification.

\section{Antibodies}

CFTR is recognized using M3A7 mouse monoclonal antibody (Millipore [Upstate] \# 05-583, 1:2000) and 217 mouse monoclonal antibody (University of North Carolina); ExoS-GAP was detected by Anti-HA mouse monoclonal antibody (Sigma MDL \# MFCD00803873; 1:5000) in immunoblots and HA.11 Clone 16B12 mouse monoclonal antibody (Covance \# MMS-101P; 1:1000) in immunohistochemical staining. Anti-mouse IgG F(ab') ${ }_{2}$ fragment-Cy3 antibody ( Sigma \# C2181; 1:250) was used as the secondary antibody in immunocytochemical analysis. GAPDH was detected with anti-GAPDH mouse monoclonal antibody (US Biological Cat. \# G8140-01; 1:10000). $\mathrm{Na}^{+} / \mathrm{K}^{+}$ATPase was detected with anti- $\mathrm{Na}^{+} / \mathrm{K}^{+}$ATPase $\alpha-1$ mouse monoclonal antibody clone C464.6 (Millipore [Upstate] \# 05-369, 1:10000). The secondary antibodies used for immunoblotting were ECL anti-mouse IgG, HRP linked monoclonal whole antibody (GE Healthcare UK limited \# NA931V; 1:30000).

\section{Cell transfection}

Cells were grown to $>90 \%$ confluence overnight and plasmids were transfected using Lipofectamine 2000 (Invitrogen Cat. \# 11668-019) according to the manufacturer's protocol. Medium was changed back to regular cell growth medium at $5 \mathrm{hrs}$. after transfection. All experiments were carried out $24 \mathrm{hrs}$. post transfection.

\section{Immunoblot analysis}

Cells were lysed at $24 \mathrm{hrs}$. post-transfection using lysis buffer (1\% deoxycholic acid, 1\% Triton X-100, $0.1 \%$ SDS, $150 \mathrm{mM} \mathrm{NaCl}, 1 \mathrm{mM}$ EDTA, $10 \mathrm{mM}$ Tris $\mathrm{HCl}$ pH 7.5 and protease inhibitor [complete protease inhibitor form Roche] added just before use) and total protein concentration was measured using a BCA assay kit (Pierce, USA). The samples were incubated at $37^{\circ} \mathrm{C}$ for $30 \mathrm{~min}$ with $5 \mathrm{X}$ loading buffer. The samples were loaded onto pre-made SDS-page gels (Biorad, USA) and electrophoresed at $125 \mathrm{~V}$. Proteins were transferred onto a PVDF membrane (Biorad \# 162-0177) at 100V for $65 \mathrm{~min}$. The PVDF membrane was blocked in 5\% non-fat milk for 2 hrs.; The appropriate primary antibody was added and the membrane was incubated overnight at $4^{\circ} \mathrm{C}$. The next day the membrane was washed with Tris-buffered saline with Tween20 (TBS-T) (0.5:1000) three times for $15 \mathrm{~min}$ each. The membrane was then incubated with appropriate secondary antibody for $1 \mathrm{hr}$. at room temperature. After three more $15 \mathrm{~min}$ washes with TBS-T, the membrane was then 
exposed with Super Signal (Pierce) and the protein density (chemiluminesence signal) was measured using Fuji Film Intelligent Dark Box LAS-3000.

\section{Surface biotinylation}

At 24 hrs. post-tranfection the cells were washed four times with ice cold PBS C/M (PBS containing $0.1 \mathrm{mM} \mathrm{CaCl}_{2}$ and $1 \mathrm{mM}$ of $\mathrm{MgCl}_{2}$ ). The cells were then incubated with $1 \mathrm{mg} / \mathrm{ml}$ sulpho-NHS-SS-biotin (Pierce Cat. \# 21331) for $1 \mathrm{hr}$. at $4^{\circ} \mathrm{C}$. The biotin was quenched with three washes of $192 \mathrm{mM}$ glycine (GibcoBRL \#15527-013) in PBS at $4^{\circ} \mathrm{C}$. The cells were lysed using lysis buffer (1\% deoxycholic acid, 1\% TritonX-100, $0.1 \%$ SDS, $150 \mathrm{mM} \mathrm{NaCl}, 1 \mathrm{mM}$ EDTA, $10 \mathrm{mM}$ Tris $\mathrm{HCl}$ [pH 7.5] and protease inhibitor added just before use) and a $50-\mu \mathrm{l}$ aliquot of protein was removed for estimation of total protein. To the remaining sample ultra link immobilized NeutrAvidin beads (Pierce Cat. \# 53150) were added and the samples were incubated at $4^{\circ} \mathrm{C}$ for $2 \mathrm{hrs}$. The samples were then washed with lysis buffer five times. The bound surface proteins were eluted from the beads with $50 \mu \mathrm{l}$ of $2 \mathrm{X}$ loading buffer by incubating at $42^{\circ} \mathrm{C}$ for $30 \mathrm{~min}$. The eluted proteins were analyzed by immunoblotting.

\section{Lysosomal inhibition studies}

At 24 hrs. post-transfection, BafilomycinA1 $(0.2 \mu \mathrm{M}$; Calbiochem) was added to test dishes and an equal amounts of DMSO was added to control dishes. Cells were incubated for $5 \mathrm{hrs}$. at $37^{\circ} \mathrm{C}$, then lysed and analyzed by immunoblotting.

\section{Proteosomal Inhibition studies}

At $24 \mathrm{hrs}$. post-transfection, MG-132 (5 $\mu \mathrm{M}$; Calbiochem) was added to test dishes and an equal amounts of DMSO was added to control dishes. Cells were incubated for 5 hrs. at $37^{\circ} \mathrm{C}$, then lysed and analyzed by immunoblotting.

\section{Immunocytochemistry}

Cells were grown on 12-well plate inserts (Falcon \#35 3180 0.4- $\mu$ m pore size) and transiently transfected with plasmid using Lipofectamine 2000. Then they were washed with ice-cold PBS C/M (C/M: $0.1 \mathrm{mM} \mathrm{CaCl}_{2}$ and $1.0 \mathrm{mM} \mathrm{MgCl}_{2}$ ) three times and fixed with $4 \%$ paraformaldehyde (Electron Microscopy Sciences \#15710 16\% solution) for 15 minutes at room temperature. The wells were then washed three times with ice cold PBS C/M and permeabilized with 0.25\% Triton X-100 (Sigma Ultra T9284) for 10 min at room temperature. The wells were again washed three times with ice-cold PBS C/M and blocked with 5\% Donkey serum (Sigma \# D9663) for $40 \mathrm{~min}$. The cells were then incubated with primary antibody against HA tag (HA.11 Clone 16B12 Monoclonal Antibody) in 2.5\% donkey serum for 40 min. The cells were then washed three times with PBS C/M for $5 \mathrm{~min}$ and incubated with secondary antibody (F(ab') fragment-Cy3 antibody) in $2.5 \%$ donkey serum for $30 \mathrm{~min}$. The cells were then washed with $2.7 \% \mathrm{NaCl}$ in PBS for 2 min, then three times for 5 min each with ice-cold PBS C/M. The filters from the inserts were cut out using a scalpel and mounted onto a glass slide and sealed with a cover slip after adding mounting antifade medium (Vectasheild with DAPI H-1200, Vector laboratories). Images were taken at 63X magnification using a Zeiss 510 Meta \#2 confocal microscope (Microscope Facility, Johns Hopkins University).

\section{Statistical analysis}

Statistical analysis was carried out using Microsoft Excel Data Analysis tool and PRISM (GraphPad version 5.0b). Two-tailed student t-test and ANOVA analysis were used with preset $\alpha<0.05$ for significance.

\section{Results}

ExoS-GAP increases the total levels of mature WT CFTR in epithelial cells

CFBE41o- cells stably expressing wild type (WT) CFTR were transiently transfected with ExoS-GAP, then lysed at $24 \mathrm{hrs}$. after transfection, and CFTR total protein levels were analyzed by immunoblotting. The cells transfected with ExoS-GAP showed an $\sim 2$ fold increase in the total mature WT CFTR levels when compared to the control cells (Fig. $1 \mathrm{~A}, \mathrm{~B}$ ). Increasing the amount of ExoS-GAP transfected resulted in a dose dependent increase in the 


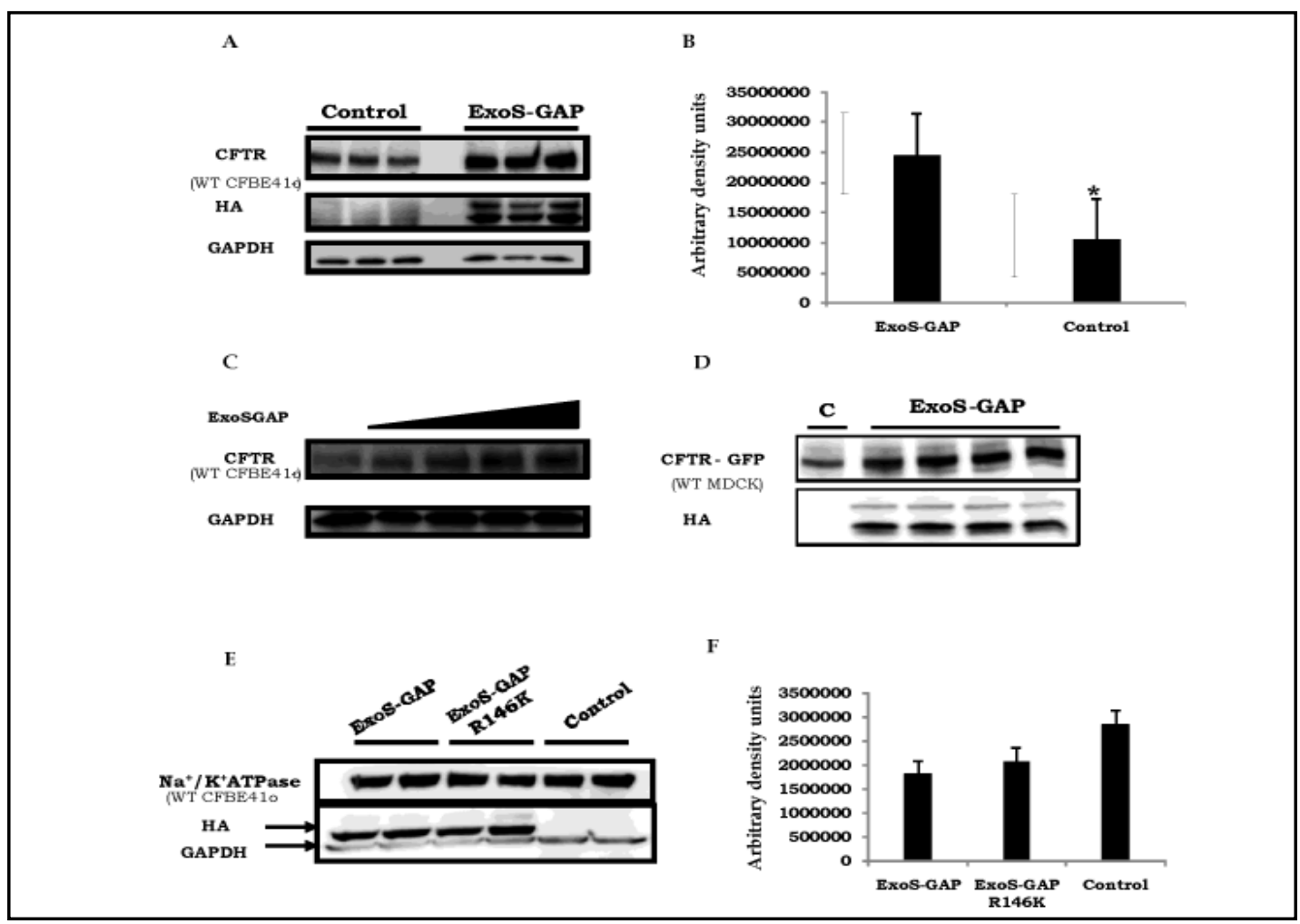

Fig. 1. Effect of ExoS on WT CFTR: A) Representative immunoblot of the total protein levels of mature CFTR in CFBE41o- cells stably expressing WT CFTR. B) Graphical representation of the mature CFTR total protein levels in arbitrary density units normalized to GAPDH. A significant increase in the total mature CFTR levels is seen in the ExoS-GAP treated cells compared to the controls. $(\mathrm{p}<0.05$, paired t-test, 2 tailed, $\mathrm{n}=15)$. C)

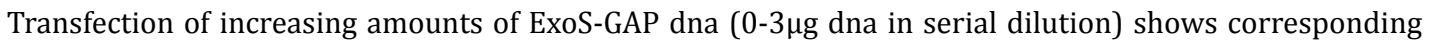
increase in the total levels of mature CFTR (n=3). D) Total protein levels of mature CFTR in MDCK cells stably expressing WT GFP-tagged CFTR $(n=3)$. E) Total protein levels of $\mathrm{Na}^{+} / \mathrm{K}^{+}$ATPase do not show significant differences between the control CFBE41o- cells and the cells treated with ExoS-GAP or ExoS-GAP R146K (a loss of function mutation of ExoS-GAP). F) Graphical representation of $\mathrm{Na}^{+} / \mathrm{K}^{+}$ATPase total protein in the 3 treatment groups ( $\mathrm{p}>0.5$, One-way ANOVA, $n=7)$. Anti-his tag $(\mathrm{HA})$ antibody used to detect ExoS-GAP and ExoS-GAP-R146K.

total levels of CFTR (Fig. $1 \mathrm{C}$ ). A similar effect on mature WT CFTR was observed in the MDCK stable cell line (Fig. 1 D).

To determine whether this effect of ExoS-GAP is specific to CFTR, we looked at the total amount of $\mathrm{Na}^{+} / \mathrm{K}^{+}$ATPase, another membrane protein. ExoS-GAP did not affect the total protein levels of $\mathrm{Na}^{+} / \mathrm{K}^{+}$ATPase (Fig. $1 \mathrm{E}, \mathrm{F}$ ) indicating that the effect of ExoS-GAP is relatively specific to the trafficking pathway of CFTR though most likely not exclusively limited to CFTR.

\section{GAP activity of ExoS is required for its effect on CFTR}

Next we wanted to determine whether the GAP activity of ExoS was responsible for the observed increases in the total protein levels of CFTR in CFBE41o- cells. The arginine residue at position 146 (R146) has been shown to be the essential residue for the GAP activity of ExoS $[5,26]$. Site-directed mutation of arginine to lysine at position 146 (R146K) results in the loss of GAP activity in ExoS. Transfection of R146K ExoS-GAP into CFBE41o- cells did not increase the total levels of mature WT CFTR when compared to the ExoS-GAP transfected cells (Fig. 2 A, B). This indicates that the GAP activity is essential and sufficient to mediate the effect of ExoS-GAP on the total levels of mature WT CFTR. 


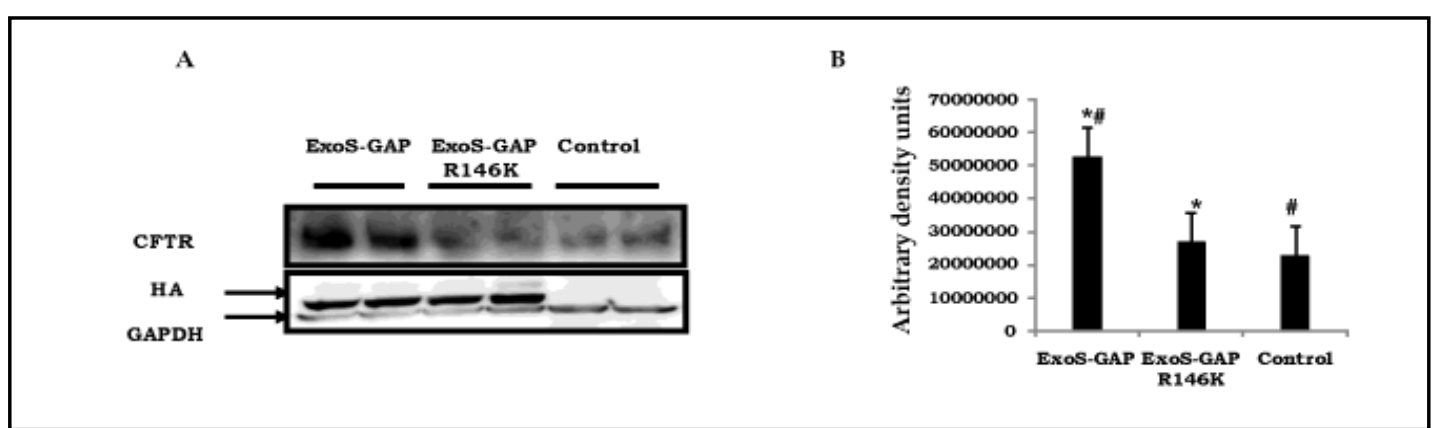

Fig. 2. Mutation of ExoS-GAP activity: A) Total protein levels of mature CFTR in CFBE41o- cells transfected with ExoS-GAP, ExoS-GAP R146K and controls. B) Graphical representation of the total protein levels of mature CFTR in arbitrary density units normalized to GAPDH $(\mathrm{p}<0.05$, paired t-test, $n=6 ; \mathrm{p}>0.05$ between R146K and controls, paired t-test, $n=6$ ).

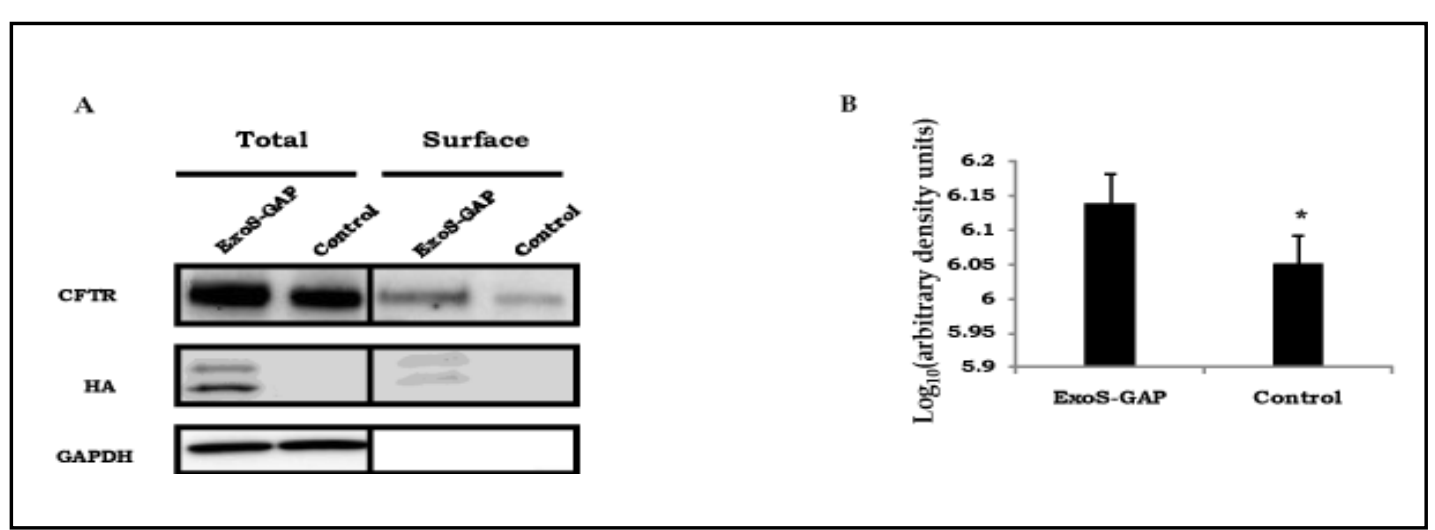

Fig. 3. Surface biotinylation studies: A) Surface levels of mature CFTR in CFBE41o- cells. B) Graphical representation of surface CFTR levels as arbitrary density units in the ExoS-GAP transfected cells compared to the controls. There is a significant increase in the available surface CFTR in ExoS-GAP transfected cells ( $\mathrm{p}<0.05$, paired t-test, $\mathrm{n}=3$ ).

\section{ExoS-GAP increases the surface availability of CFTR}

The surface availability of CFTR ultimately determines the extent of its role in the disease process. We determined if an increase in the total levels of mature WT CFTR translated into a corresponding increase in the surface availability of CFTR. ExoS-GAP was transiently transfected into CFBE 41o- stable cells, and the surface amounts of CFTR was estimated at 24 hrs. post-transfection by surface biotinlylation. The cells transfected with ExoS-GAP showed significantly elevated levels of surface protein when compared to the control cells (Fig. $3 \mathrm{~A}$, B).

\section{ExoS-GAP does not alter the plasma membrane trafficking or localization of CFTR}

Since ExoS-GAP is known to target Rabs that are also involved in the CFTR trafficking pathways, our next step was to determine whether ExoS-GAP affected CFTR trafficking resulting in protein mislocalization. Immunohistochemical analysis of MDCK cells stably expressing GFP-tagged CFTR was carried out at $24 \mathrm{hrs}$. post-transfection with confocal microscopy. No differences in the localization pattern of CFTR in the ExoS-GAP transfected cells were seen when compared to the control cells or the R146K mutant transfected cells (Fig. 4 A, B). This suggests that ExoS-GAP does not alter the trafficking of CFTR to the plasma membrane, nor does the increased CFTR protein form intracellular aggregates. Also, the overall level of GFP fluorescence in the ExoS-GAP transfected cells was higher than that in the control or the R146K mutant transfected cells (Fig. 4 A, B) consistent with the increase in 
A

ExoS-GAP

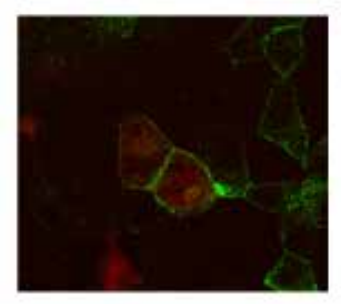

DAPI
Control

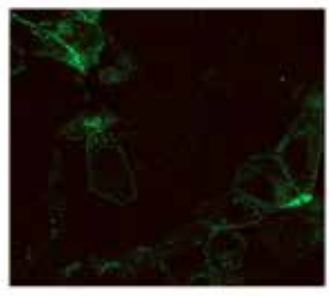

AntiHa
B

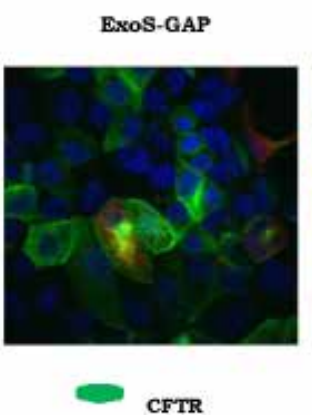

ExoS-GAP R146K

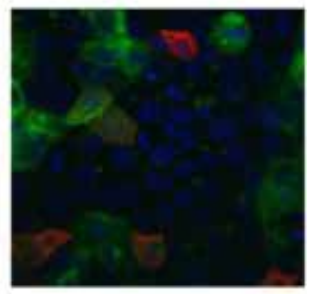

Fig. 4. Immunohistochemical data: A) Localization pattern of CFTR (green) in the ExoS-GAP transfected cells compared to localization pattern of the control cells. There are no differences in the localization pattern between the 2 groups. B) Localization pattern and field brightness of ExoS-GAP treated cells compared to ExoS-GAP R146K mutant. The ExoS-GAP transfected cells have brighter CFTR expressing fields when compared to the controls or the mutant transfected cells.

total and surface levels of CFTR observed following ExoS-GAP transfection in the immunoblot analysis.

ExoS-GAP affects total CFTR levels by inhibiting the lysosomal degradation of CFTR

Cellular increases in total protein levels are attributable either to stimulation of protein synthesis or inhibition of protein degradation. The known molecular interactions between ExoS and SMWGP led us to hypothesize that ExoS-GAP was targeting the degradation of CFTR. Since most of the mature WT CFTR is trafficked to the lysosome for degradation, we tested our hypothesis by inhibiting lysosomal degradation with BafilomycinA1. BafilomycinA1 is a well-known V-ATPase inhibitor that disrupts lysosomal $\mathrm{pH}$ and hence lysosomal function. CFBE41o- cells were treated for $5 \mathrm{hrs}$. with $0.2 \mu \mathrm{M}$ BafilomycinA1 at $24 \mathrm{hrs}$. post-transfection, following which the cells were lysed and analyzed for CFTR total protein levels. Treating the control cells with BafilomycinA1 resulted in an increase in the total protein levels of CFTR as a result of CFTR degradation inhibition (Fig. 5 A). However, the ExoS-GAP transfected cells showed no increase in the total protein levels of CFTR following BafilomycinA1 treatment (Fig. 5 A). The amount of increase in mature protein levels of CFTR in cells treated with BafilomycinA1 over the protein levels in cells treated with DMSO (BafilomycinA1 vehicle) was significantly higher in the control cells when compared to the ExoS-GAP transfected cells (Fig. 5 B). This result indicates that ExoS-GAP inhibits trafficking of CFTR to the lysosome thereby decreasing its degradation and increasing its total protein levels. This inhibition of the degradation pathway by ExoS-GAP was not enhanced by BafilomycinA1 mediated lysosomal inhibition ruling out additive effect.

To further investigate this process, we evaluated the clearance of CFTR following inhibition of protein synthesis. These experiments were carried out in MDCK cells stably expressing GFP-tagged CFTR because of the faster clearance rate of CFTR in MDCK cells compared to CFBE410- cells stably expressing CFTR (unpublished data). Transiently transfected MDCK cells stably expressing GFP-tagged CFTR were treated with $25 \mu \mathrm{g} / \mathrm{ml}$ cycloheximide for $2.5 \mathrm{hrs}$. then samples were collected every $30 \mathrm{~min}$. The total protein levels of mature CFTR at 2.5 hrs. post-cycloheximide treatment were more stable in the ExoS-GAP transfected cells than in the control or R146K mutant transfected cells (Fig. 5 C) corroborating the earlier observation that ExoS-GAP activity inhibited the lysosomal degradation of CFTR. The total mature CFTR protein levels in the ExoS-GAP transfected cells were not significantly different after $2.5 \mathrm{hrs}$. of treatment with cycloheximide alone or combined cycloheximide and BafilomycinA1 treatment (Fig. 5 D). Furthermore, the mature 
$\mathbf{A}$

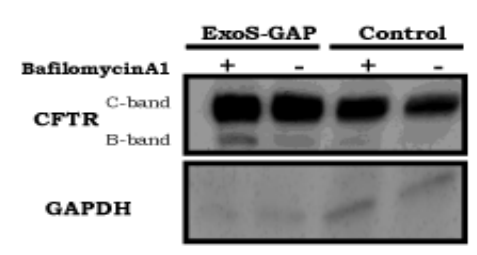

B

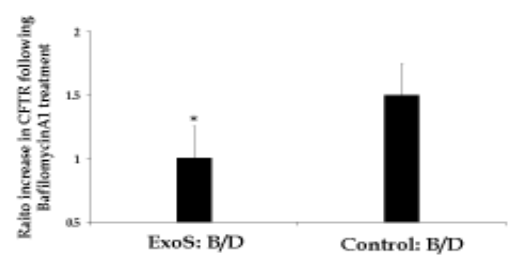

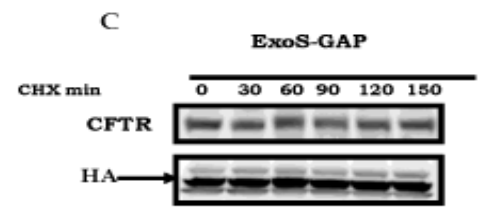

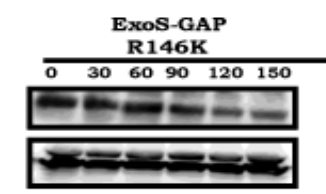

Control

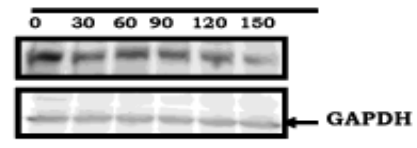

D

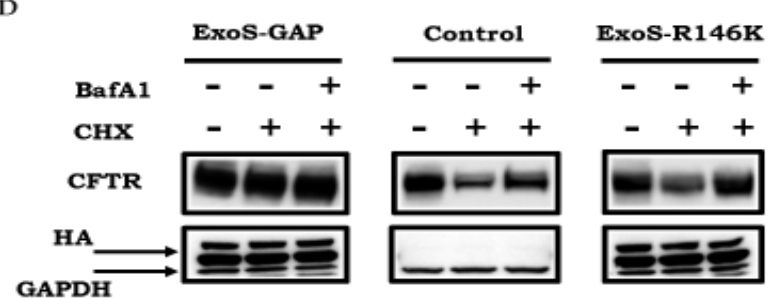

Fig. 5. Lysosomal degradation studies: A) Mature CFTR (C-band) protein levels in the CFBE41o- ExoS-GAP transfected and control cells treated with $0.2 \mu \mathrm{M}$ BafilomycinA1. B) Graphical representation of increase in mature CFTR in BafilomycinA1 treated cells (B) over DMSO treated cells (D) expressed as a ratio ( $p<0.05$, paired t-test, $n=3$ ). C) Clearance of CFTR following treatment with cycloheximide (CHX) $25 \mu \mathrm{g} / \mathrm{ml}$ over 150 min in the ExoS-GAP and ExoS-GAP R146K transfected cells compared to the controls. CFTR is more stable in the ExoS-GAP transfected cells when compared to the controls or ExoS-GAP R146K transfected cells ( $n=4)$. D) CFTR turnover with CHX and CHX+BafilomycinA1. BafilomycinA1 pre-treatment stabilized mature CFTR levels in the control and ExoS-GAP R146K transfected cells. (n=3; B/D: Bafilomycin treated cells/DMSO treated cells).

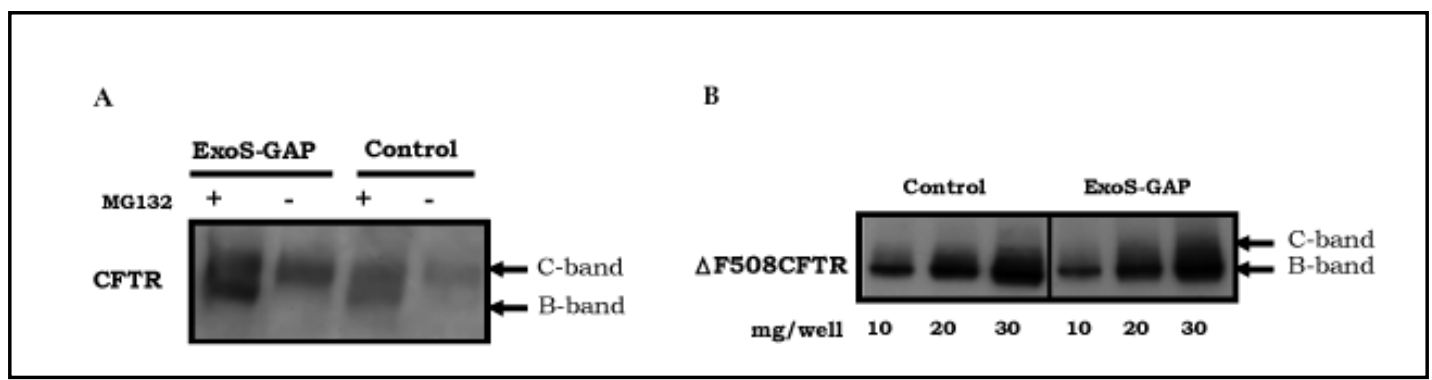

Fig. 6. Proteasomal degradation studies: A) Total mature CFTR (C-band) and immature CFTR (B-band) levels following treatment with $5 \mu \mathrm{M}$ MG-132. Equal amounts of total protein increase in mature and immature CFTR is seen with MG-132 treatment in both the ExoS-GAP transfected cells and the control cells $(\mathrm{n}=3)$. B) CFTR total protein levels in CFBE41o- cells stably expressing $\triangle$ F508CFTR show no differences in the control and ExoS-GAP transfected cells $(n=3$; each sample is loaded in increasing amounts of total protein: $10,20,30 \mathrm{mg} /$ well to rule out the effect of protein saturation on observed results).

CFTR protein levels in the control and the R146K mutant transfected cells were rescued from clearance by cycloheximide after 5 hrs. of pre-treatment with BafilomycinA1 (Fig. 5 D). These observations demonstrate that ExoS-GAP upregulates the total protein levels of CFTR by inhibiting its lysosomal degradation. 

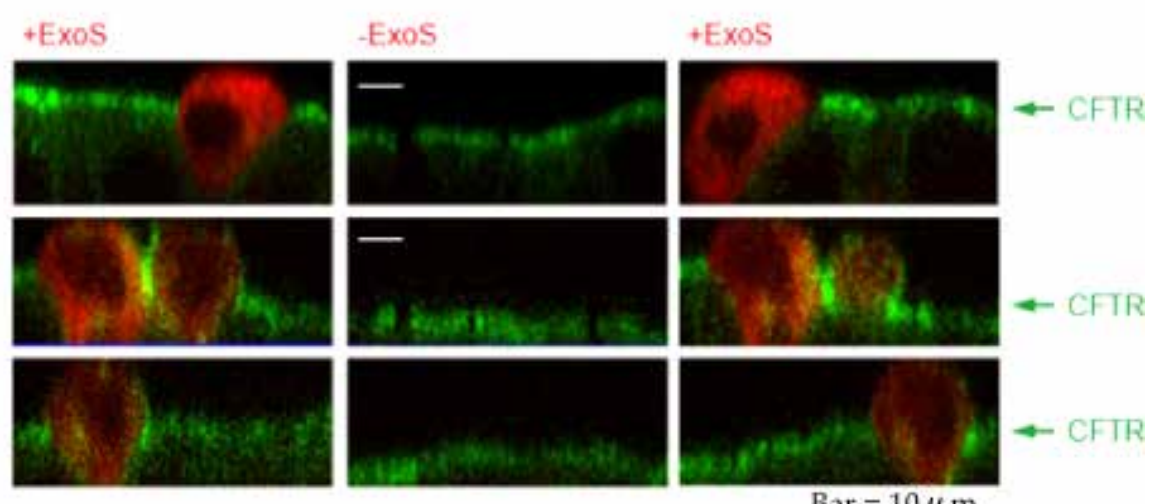

Bar $=10 \mu \mathrm{m}$

Fig. 7. Cell dissociation: Z-section confocal images of MDCK cells transfected with ExoS-GAP. Cells expressing large quantities of ExoS-GAP lie above the apical membrane plane of the polarized MDCK cell monolayer.

\section{ExoS-GAP does not affect the ER associated proteasomal degradation of CFTR}

The immature WT CFTR (B-band) is trafficked to the proteasomes for degradation [27]. In order to determine whether ExoS-GAP affects the ER associated degradation (ERAD) of CFTR, we treated transiently transfected CFBE41o- cells with $5-\mu \mathrm{M}$ MG-132, a known protesomal inhibitor. In the control cells, as expected, an increase in both the mature (C-band) and immature (B-band) forms of CFTR was observed (Fig. 6 A). An equivalent increase in the mature and immature forms of CFTR was observed in the ExoS-GAP transfected cells (Fig. 6 A). This result indicates that ExoS-GAP does not inhibit the proteasomal degradation of CFTR. This observation was corroborated by showing that ExoS-GAP failed to increase the total protein levels of $\triangle$ F508 CFTR in CFBE41o- cells stably expressing $\triangle$ F508 CFTR (Fig. 6 $\mathrm{B}$ ), a misfolding mutant of CFTR that is predominantly targeted to ERAD [28]. Taken together with the observation that ExoS-GAP increased the total protein levels of the mature form of CFTR, these findings demonstrate that effects of ExoS-GAP on CFTR are localized to the postGolgi processing of CFTR.

\section{ExoS-GAP induces cell-cell dissociation in epithelial cells}

An interesting ancillary finding during our immunohistochemical study of MDCK cells stably expressing GFP-tagged CFTR was the identification of cell-cell dissociation in cells expressing a significant amount of ExoS-GAP. This phenomenon was seen as dissociated cells appearing above the apical plane of the cell monolayer in $\mathrm{Z}$ sections of the immunohistochemical confocal microscopy data (Fig. 7). This dissociation was not observed in cells expressing mild-to-moderate amounts of ExoS-GAP. However, if this resulted in a functionally significant disruption in the epithelial barrier was not evaluated. A recent study by Soong et al. shows that the GAP activity of ExoS does not contribute to the epithelial barrier disruption caused by ExoS [29]. The significance of cell-cell dissociation induced by ExoS-GAP needs further evaluation. The possible pathophysiological implications of this phenomenon are briefly addressed in the discussion.

\section{Discussion}

This study demonstrates that the GAP activity of ExoS increases the total and surface protein levels of the mature form of WT CFTR in bronchial epithelial cells. We have shown that the increase in mature WT CFTR protein is a result of decreased trafficking of CFTR to the lysosome. Based on the known molecular targets of ExoS, we speculate that the most 
likely mechanism by which ExoS-GAP decreases the lysosomal degradation of CFTR is by inactivating Rab7 which is involved in the lysosomal trafficking of CFTR or Rab9 which is involved in trafficking of CFTR to trans-Golgi [10]. ExoS is known to co-immunoprecipitate with Rab9 [30] but physical interaction between ExoS and Rab7 has not been examined. Identification of these interactions was beyond the scope of this study. Prior studies of epidermal growth factor (EGF) induced EGF receptor (EGFR) degradation have shown that ExoS decreases the lysosomal degradation of EGFR [31]. However, it is the ADPRT activity of ExoS that results in decreased trafficking of EGFR to the lysosome [31]. In our study, we have used a full-length construct of ExoS with its ADPRT activity mutated (ExoS-E381DHA). Hence, we can reasonably conclude that the effects seen on CFTR are predominantly mediated by its GAP activity. Also, inactivation of the GAP activity of the ExoS-GAP (ExoS-GAP R146K) resulted in the abolition of its effects on WT CFTR mature total protein levels.

It is curious to note that the same toxin affects the trafficking of the two membrane proteins, CFTR and EGFR, to the lysosomes via different enzymatic activities. This phenomenon may indicate that membrane proteins targeted to the same molecular compartment have subtle differences in trafficking. This difference is further underscored by the observation that ExoS-GAP did not increase the total protein levels of $\mathrm{Na}^{+} / \mathrm{K}^{+} A T P a s e ; a$ membrane protein that traffics to the lysosome for degradation [32]. We have not identified the exact molecular pathway targeted by ExoS-GAP that traffics CFTR to the lysosome. Based on the current understanding of the nature of ExoS-GAP, it is likely that SMWGP are being targeted by ExoS-GAP [30]. If this hypothesis were to be correct, it is likely that other protein trafficked through these pathways are regulated by ExoS-GAP and may contribute to the toxicity of ExoS-GAP. Also, even though we have shown that inhibition of lysosomal degradation is the underlying mechanism by which ExoS-GAP increases the WT CFTR mature total protein levels, we acknowledge that the increase could also be mediated by ExoS-GAP induced release of soluble factors acting via autocrine or paracrine signaling. Further experimentation is required to elucidate if cis- or trans-regulation is a parallel process occurring in ExoS-GAP expressing epithelial cells.

The effect of ExoS-GAP on CFTR provides insight into understanding some of the pathological findings seen following PA infections. It has previously been documented that surface expression of CFTR in mouse bronchial epithelium is enhanced by PA infection [13]. We propose that the ExoS-GAP mediated decrease in mature WT CFTR degradation explains the enhanced bronchial epithelial surface expression observed following PA infections in mice. CFTR is a known receptor for PA [13]. The first extra cellular loop region of CFTR from 108-117 amino acids binds PA resulting in intracellular uptake of PA into epithelial cells [13]. This intracellular uptake can be hypothesized as an advantage to both the host and the bacteria. In case of the host, CFTR-mediated cellular uptake of PA has been postulated to be an important mechanism for clearing the organism from airway surface epithelium by promoting intracellular uptake. In case of the bacteria, ExoS promotes the cellular uptake of PA possibly facilitating bacterial invasion/transmigration.

The occurrence and severity of an infectious disease is dependent on the invasive ability of the infecting agent and effectiveness of the host immune system. In case of pulmonary PA infections, enhancing the surface expression of CFTR appears to be one of the mechanisms facilitating its clearance[33]. This mechanism could explain why PA lung infections are fatal in hospitalized/ immunocompromised hosts and not clinically significant in immunecompetent hosts. In a host with a compromised immune system, PA's access to the host blood provides an ideal milieu for bacterial survival and propagation, resulting in septicemia. However, in a host with an intact immune system, the initial bacteremia is most likely prevented from progressing onto septicemia; hence there is no clinically significant disease. This leads to the possibility that identifying agents that can block the interaction of PA with CFTR or specifically inhibit the GAP activity of ExoS may help prevent bacterial transmigration and dissemination. These agents may prove to be valuable as adjuvants to antibacterial therapy in the management of Pseudomonal pneumonias. 
Also, the hypothesis that CFTR is the receptor facilitating the uptake and clearance of PA in normal individuals could explain the chronic nature of PA infection in CF patients. $\triangle$ F508CFTR (the CFTR mutation accounting for $>70 \%$ of CF in Caucasian population [10]) has a lower surface availability and decreased surface clearance of the organism [33, 34]. In our study, ExoS-GAP did not increase the total protein levels of $\triangle$ F508CFTR; possibly contributing to the ineffective clearance of PA during infection in CF. The increased intrabronchial bacterial load in conjunction with impaired mucosal clearance (resulting from reduced airway surface fluid production) provides for an ideal milieu for chronic PA infection.

Recent studies have shown that the ADPRT activity of ExoS disrupts the epithelial cell barrier [35]. The authors suggest this as the mechanism of PA invasion and dissemination. Previous studies have shown that the GAP activity of ExoS results in actin-cytoskeletal dissociation in $\mathrm{CHO}$ cells and fibroblasts causing cell rounding [7]. In the present study we have shown for the first time that cells expressing excess ExoS-GAP dissociate from the surrounding cells in the epithelial monolayer. Currently, we do not completely understand the significance of this finding. We hypothesize that the cellular dissociation could contribute towards the clearance of epithelial cells loaded with PA. If so, it suggests a process by which the host utilizes the bacterial machinery to supplement its innate immunity: first, by enhancing PA uptake by the epithelial cells via CFTR upregulation and second, by promoting the clearance of bacteria-loaded cells by causing cell-cell dissociation.

It has been suggested that the high incidence of $\Delta$ F508CFTR mutation in Caucasian population is a result of natural selection [36]. The proposed selection process has been speculated to involve survival advantage against bacterial infections like Salmonella typhi (typhoid) and Vibrio cholera (cholera) causing CFTR mediated diarrhea [36, 37]. Although the CFTR mediated diarrhea can cause fatal dehydration in case of cholera, this is not true in case of Salmonella. In Salmonella infections, the fatal nature of the disease is secondary to the bacterial transmigration to the blood stream. It has been shown that WT CFTR promotes transmigration of S. Typhi from colonic mucosa to the submucosa in mice and T84 colonic epithelia cells [38]. Interestingly, like PA, S. typhi expresses TTST [39]. Among the S. typhi TTST, SptP has GAP activity [40]. It would be of interest to see if SptP upregulates total CFTR protein levels. If it does, it would be reasonable to hypothesize that a TTST-GAP mediated increase in CFTR facilitates bacteremia and hence severe to fatal disease process.

In summary, we have shown that the GAP activity of ExoS increases the total protein levels and surface availability of mature WT CFTR. We have also shown that one of the mechanisms for the increase is ExoS-GAP mediated inhibition of its lysosomal degradation. We propose that this enhanced CFTR expression contributes to the occurrence and severity of Pseudomonas pneumonia in hospitalized and immunocompromised patients by facilitating migration of PA into the blood stream. Therefore, agents that can block the interaction of PA with CFTR may provide a new adjuvant treatment strategy to the current conventional management of Pseudomonas pneumonia.

\section{Abbreviations}

CFTR (cystic fibrosis transmembrane conductance regulator); PA (Pseudomonas aueroginosa); GAP (GTPase activating protein); ADPRT (ADP rybosyl transferase activity); SMWGP (small molecular weight G-proteins).

\section{Acknowledgements}

We thank Dr. Dieter Gruenert (California Pacific Medical Center Research Institute) for generously providing us the CFBE410- cell lines stably expressing WT CFTR; Dr. Bruce A. Stanton (Dartmouth-Hitchcock Medical Center), and Dr. Garry Cutting (Johns Hopkins University School of Medicine) for generously providing us the MDCK cell lines stably 
Tukaye/Kwon/Guggino: ExoS-GAP Upregulates WT CFTR

expressing GFP-tagged CFTR; Dr. Joseph T. Barberi (Medical College of Wisconsin) for generously providing us the full-length ExoS-E381D-HA plasmid; Dr. J.P. Clancy (University of Alabama) for generously providing us the CFBE41o- cell lines stably expressing WT and $\triangle$ F508CFTR. We thank Dr. Deborah McClellan and Dr. Kiran Francis Rajneesh for editorial assistance. This work was supported by The Cystic Fibrosis Foundation Research Development Programs (to W. B. G.) and National Institutes of Health Grant (NHLBI) HL-47122 and (NIDDK) DK072084 (to W. B. G.).

\section{References}

-1 Sadikot RT, Blackwell TS, Christman JW, Prince AS: Pathogen-host interactions in pseudomonas aeruginosa pneumonia. Am J Respir Crit Care Med 2005;171:1209-1223.

$>2$ Moore NM, Flaws ML: Epidemiology and pathogenesis of pseudomonas aeruginosa infections. Clin Lab Sci 2011;24:43-46.

-3 Bonifacio SL, Kitterman JA, Ursell PC: Pseudomonas pneumonia in infants: An autopsy study. Hum Pathol 2003;34:929-938.

4 Barbieri JT: Pseudomonas aeruginosa exoenzyme s, a bifunctional type-III secreted cytotoxin. Int J Med Microbiol 2000;290:381-387.

5 Pederson KJ, Pal S, Vallis AJ, Frank DW, Barbieri JT: Intracellular localization and processing of pseudomonas aeruginosa exos in eukaryotic cells. Mol Microbiol 2000;37:287-299.

-6 Henriksson ML, Sundin C, Jansson AL, Forsberg A, Palmer RH, Hallberg B: Exoenzyme s shows selective adp-ribosylation and gtpase-activating protein (gap) activities towards small gtpases in vivo. Biochem J 2002;367:617-628.

7 Sun J, Barbieri JT: Exos rho gtpase-activating protein activity stimulates reorganization of the actin cytoskeleton through rho gtpase guanine nucleotide disassociation inhibitor. J Biol Chem 2004;279:4293642944.

-8 Krall R, Sun J, Pederson KJ, Barbieri JT: In vivo rho gtpase-activating protein activity of pseudomonas aeruginosa cytotoxin exos. Infect Immun 2002;70:360-367.

$>9$ Gentzsch M, Chang XB, Cui L, Wu Y, Ozols VV, Choudhury A, Pagano RE, Riordan JR: Endocytic trafficking routes of wild type and deltaf508 cystic fibrosis transmembrane conductance regulator. Mol Biol Cell 2004; $15: 2684-2696$.

10 Guggino WB, Stanton BA: New insights into cystic fibrosis: Molecular switches that regulate cftr. Nat Rev Mol Cell Biol 2006;7:426-436.

11 Pilewski JM, Frizzell RA: Role of cftr in airway disease. Physiol Rev 1999;79:S215-255.

12 Gosselin D, Stevenson MM, Cowley EA, Griesenbach U, Eidelman DH, Boule M, Tam MF, Kent G, Skamene E, Tsui LC, Radzioch D: Impaired ability of cftr knockout mice to control lung infection with pseudomonas aeruginosa. Am J Respir Crit Care Med 1998;157:1253-1262.

13 Pier GB: Role of the cystic fibrosis transmembrane conductance regulator in innate immunity to pseudomonas aeruginosa infections. Proc Natl Acad Sci USA 2000;97:8822-8828.

14 Schroeder TH, Lee MM, Yacono PW, Cannon CL, Gerceker AA, Golan DE, Pier GB: Cftr is a pattern recognition molecule that extracts pseudomonas aeruginosa lps from the outer membrane into epithelial cells and activates nf-kappa b translocation. Proc Natl Acad Sci USA 2002;99:6907-6912.

-15 Ader F, Le Berre R, Faure K, Gosset P, Epaulard O, Toussaint B, Polack B, Nowak E, Viget NB, Kipnis E, Guery BP: Alveolar response to pseudomonas aeruginosa: Role of the type iii secretion system. Infect Immun 2005;73:4263-4271.

16 Fontelo P: Protection against cholera. Science 1995;267:440.

17 Pier GB, Grout M, Zaidi T, Meluleni G, Mueschenborn SS, Banting G, Ratcliff R, Evans MJ, Colledge WH: Salmonella typhi uses cftr to enter intestinal epithelial cells. Nature 1998;393:79-82.

18 Ben Haj Khalifa A, Moissenet D, Vu Thien H, Khedher M: [virulence factors in pseudomonas aeruginosa: Mechanisms and modes of regulation]. Ann Biol Clin (Paris) 2011;69:393-403. 


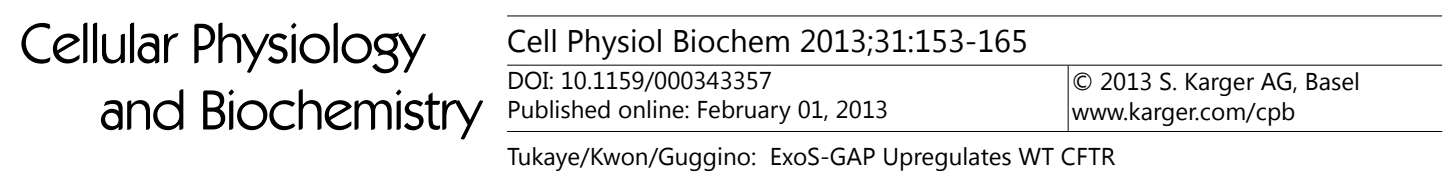

19 Bleves S, Viarre V, Salacha R, Michel GP, Filloux A, Voulhoux R: Protein secretion systems in pseudomonas aeruginosa: A wealth of pathogenic weapons. Int J Med Microbiol 2010;300:534-543.

-20 Engel J, Balachandran P: Role of pseudomonas aeruginosa type III effectors in disease. Curr Opin Microbiol 2009;12:61-66.

-21 Feltman H, Schulert G, Khan S, Jain M, Peterson L, Hauser AR: Prevalence of type III secretion genes in clinical and environmental isolates of pseudomonas aeruginosa. Microbiology 2001;147:2659-2669.

22 Nicas TI, Iglewski BH: Contribution of exoenzyme S to the virulence of pseudomonas aeruginosa. Antibiot Chemother 1985;36:40-48.

-23 Nicas TI, Frank DW, Stenzel P, Lile JD, Iglewski BH: Role of exoenzyme s in chronic pseudomonas aeruginosa lung infections. Eur J Clin Microbiol 1985;4:175-179.

24 Nicas TI, Iglewski BH: The contribution of exoproducts to virulence of pseudomonas aeruginosa. Can J Microbiol 1985;31:387-392.

25 Barbieri JT, Sun J: Pseudomonas aeruginosa exos and exot. Rev Physiol Biochem Pharmacol 2004;152:7992.

26 Goehring UM, Schmidt G, Pederson KJ, Aktories K, Barbieri JT: The n-terminal domain of pseudomonas aeruginosa exoenzyme $\mathrm{s}$ is a GTPase-activating protein for rho GTPases. J Biol Chem 1999;274:3636936372.

27 Belcher CN, Vij N: Protein processing and inflammatory signaling in cystic fibrosis: Challenges and therapeutic strategies. Curr Mol Med 2010;10:82-94.

28 Ahner A, Nakatsukasa K, Zhang H, Frizzell RA, Brodsky JL: Small heat-shock proteins select deltaf508-cftr for endoplasmic reticulum-associated degradation. Mol Biology Cell 2007;18:806-814.

29 Soong G, Parker D, Magargee M, Prince AS: The type III toxins of pseudomonas aeruginosa disrupt epithelial barrier function. J Bacteriol 2008;190:2814-2821.

-30 Zhang Y, Deng Q, Barbieri JT: Intracellular localization of type III-delivered pseudomonas exos with endosome vesicles. J Biol Chem 2007;282:13022-13032.

31 Deng Q Barbieri JT: Modulation of host cell endocytosis by the type III cytotoxin, pseudomonas exos. Traffic 2008;9:1948-1957.

-32 Lecuona E, Sun H, Vohwinkel C, Ciechanover A, Sznajder JI: Ubiquitination participates in the lysosomal degradation of Na,K-ATPase in steady-state conditions. Am J Respir Cell Mol Biol 2009;41:671-679.

-33 Pier GB, Grout M, Zaidi TS: Cystic fibrosis transmembrane conductance regulator is an epithelial cell receptor for clearance of pseudomonas aeruginosa from the lung. Proc Natl Acad Sci USA 1997;94:1208812093.

-34 Schroeder TH, Reiniger N, Meluleni G, Grout M, Coleman FT, Pier GB: Transgenic cystic fibrosis mice exhibit reduced early clearance of pseudomonas aeruginosa from the respiratory tract. J Immunol 2001;166:74107418.

35 Bridge DR, Martin KH, Moore ER, Lee WM, Carroll JA, Rocha CL, Olson JC: Examining the role of actinplasma membrane association in pseudomonas aeruginosa infection and $\mathrm{t} 3 \mathrm{~s}$ translocation in migratory $\mathrm{t} 24$ epithelial cells. Infect Immun 2012;80:3049-3064.

36 Guggino SE: Evolution of the delta F508 CFTR mutation. Trends Microbiol 1999;7:55-56; discussion 56-58.

37 Alfonso-Sanchez MA, Perez-Miranda AM, Garcia-Obregon S, Pena JA: An evolutionary approach to the high frequency of the delta F508 CFTR mutation in european populations. Med Hypotheses 2010;74:989-992.

-38 Lyczak JB, Zaidi TS, Grout M, Bittner M, Contreras I, Pier GB: Epithelial cell contact-induced alterations in salmonella enterica serovar typhi lipopolysaccharide are critical for bacterial internalization. Cell Microbiol 2001;3:763-772.

-39 Winstanley C, Hart CA: Type III secretion systems and pathogenicity islands. J Med Microbiol 2001;50:116126.

40 Aktories K, Schmidt G, Just I: Rho GTPases as targets of bacterial protein toxins. Biol Chem 2000;381:421426. 